\title{
FPGA-Based Upgrade of the Read-Out Electronics for the Low Energy Polarimeter at COSY/Jülich
}

\author{
Nils HEMPELMANN*广 \\ Forschungszentrum Jülich GmbH \\ E-mail: n. hempelmannefz-juelich.de
}

The low energy polarimeter (LEP [1]) is a polarimeter in the injection beam line of the Cooler Synchrotron (COSY [2]). COSY is a facility for cooled polarized beams at the Forschungszentrum in Jülich. The beam polarization is measured using scattering off carbon and polyethylene $\left(\mathrm{CH}_{2}\right)$ targets. Up to now only elastic scattering off the carbon atoms is used for polarimetry. The outgoing particles are detected using twelve plastic scintillators installed in groups of three to the left, to the right, above, and below the beam.

LEP is the routine tool for beam set-up, but limited in performance. The current read-out electronics consists of analog NIM modules. It can either be used to measure pulse height spectra at a data rate limited to about $50 \mathrm{kHz}$ or to merely count the pulses within an adjustable range in amplitude, which allows for a faster measurement. A further motivation for the upgrade is the missing ability to determine the tensor polarization $P_{z z}$.

A new system using analog pulse sampling and an FPGA chip for signal processing was installed and tested. The ejectile particles were identified by relative time of flight measurement using a signal from the RF amplifier of the cyclotron used for acceleration as a reference. The new system is able to measure the time at which a particle arrives to an accuracy in the order of $50 \mathrm{ps}$. The presentation includes a review of available systems and the report about first measurements in May 2015.

XVIth International Workshop in Polarized Sources, Targets, and Polarimetry

14-18 September 2015

Ruhr-University Bochum, Germany

* Speaker.

${ }^{\dagger}$ On behalf of the JEDI-Collaboration 


\section{Low Energy Polarimeter at COSY}

The low energy polarimeter (LEP) [1] is a polarimeter in the injection beam line at the Cooler Synchrotron (COSY [2]). COSY is a storage ring for polarized protons and deuterons. The polarized particles are generated by a colliding beam source and then preaccelerated in a cyclotron to a kinetic energy of $45 \mathrm{MeV}$ for protons or $75 \mathrm{MeV}$ for deuterons. LEP measures the beam polarization before the particles are injected into the main ring.

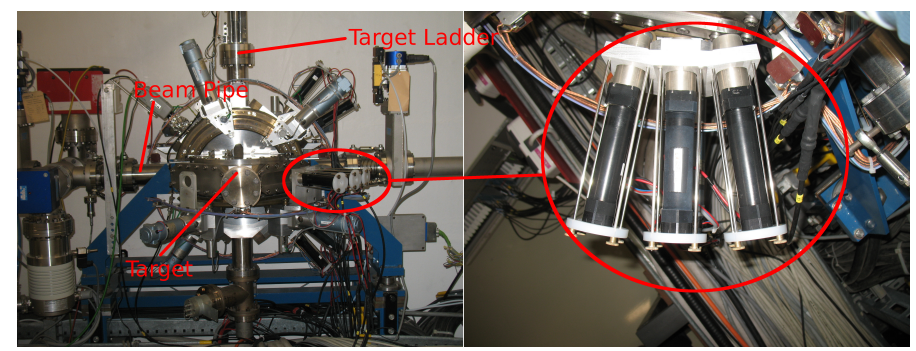

Figure 1: Picture of the Low Energy Polarimeter (left) and close-up on a group of particle detectors (right).

Fig. 1 shows a photo of LEP. Particles enter through the beam pipe and are scattered off a target at the center. The target can be selected using a ladder-like construction. Carbon and polyethylene foil targets with a thickness between $0.1 \mathrm{~mm}$ and $0.5 \mathrm{~mm}$ are available. The scattered particles are detected by twelve plastic scintillators with photomultiplier readout. The detectors are mounted in groups of three (fig. 1, right), spaced $10^{\circ}$ apart. One such group is positioned in each cardinal direction of scattering (left, right, up and down). The detector groups can be rotated around the target as a rigid unit to select a scattering angle.

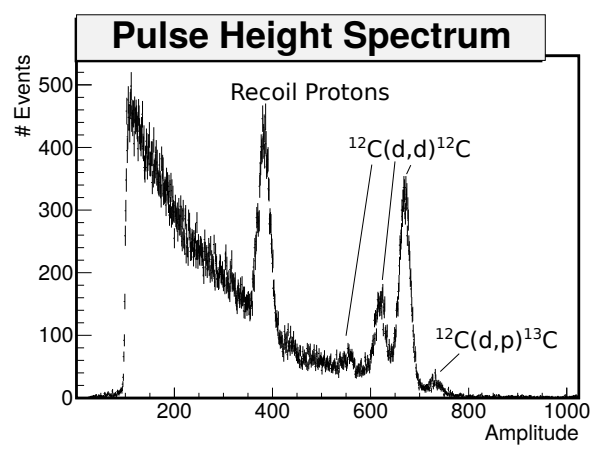

(a) Pulse height spectrum

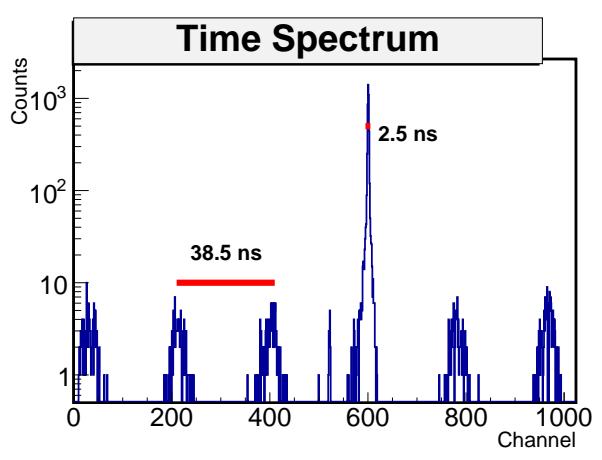

(b) Coincidence measurement

Figure 2: Event selection in the old read-out electronics. Left: Amplitude spectrum of deuterons scattered off a $\mathrm{CH}_{2}$-target. Right: Time between recoil and ejectile detection. The resolution is about $2.5 \mathrm{~ns}$. The smaller peaks correspond to the cyclotron frequency.

The current read-out electronics consists of analog NIM modules. The elastic scattering events used for polarimetry are selected using pulse height discrimination. Fig. 2a shows a pulse height spectrum of deuteron- $\mathrm{CH}_{2}$ scattering measured using the old electronics. The single peak on the 
left corresponds to recoil protons from the target, the peaks on the right correspond to elastic and inelastic carbon scattering. In addition to the pulse height discrimination, the electronics can measure deuterons and recoil protons in coincidence. Fig. $2 \mathrm{~b}$ shows a plot of the time between the detection of recoil and ejectile measured using a time-to-amplitude converter. The resolution is about $2.5 \mathrm{~ns}$. The smaller peaks in the time spectrum are random coincidences corresponding to the cyclotron frequency. The two detected particles can come from different cyclotron pulses, which means that the time between their detection is an integer multiple of the cyclotron period.

\section{Readout Electronics}

The main aim of the upgrade is to facilitate a better online measurement of the beam polarization by increasing the measurable data rate to a value in the order of $1 \mathrm{MHz}$. The detectors are read-out using a GANDALF module [3], which provides an eight-channel ADC and an FPGA for signal processing. The sampling rate is $1 \mathrm{GHz}$ at a resolution of 12 bit. Seven of the eight ADC channels can be connected to detectors, the eighths is connected to a signal from the RF amplifier of the cyclotron. This signal can be used as a reference for time of flight measurements. An absolute measurement is not possible as the precise signal run times and the time of flight from the cyclotron to the polarimeter are not known, which adds an unknown constant offset to each channel.

The firmware of the module had to be modified for this experiment. The central part of the firmware is a constant fraction discriminator (CFD) that was already implemented in the original version. The CFD detects pulses in the input voltage and calculates their time and amplitude. The time measurement has a precision of about 50 ps. The time of flight is the difference in time between each pulse from the detectors and the previous cyclotron pulse. Pulses whose amplitude and time of flight lie within an adjustable range are counted to determine the event rates in each detector, which are later used to calculate the asymmetry. These count rates are periodically transferred to a computer via an USB port. In addition to the count rates the full event information (time and amplitude) is written for a prescaled subset of events to calibrate the detector. The wave form of the pulses can also be read-out with a prescaler.

\section{First Measurements}

During the beam time in the summer of 2015 the first measurements with the new read-out electronics were taken. The experiments during this beam time used polarized deuterons. The measurements with the polarimeter included amplitude and time spectra as well as the beam polarization over time. After the main beam time, some additional measurements using unpolarized protons were taken.

Fig. 3 shows the main results of the deuteron-carbon scattering experiments. Fig. 3a shows a plot of the amplitude and the time of flight. As these quantities are connected by kinematics, one would expect each type of particle to form a single line in the plot. In the observed spectrum there are multiple lines corresponding to each type of particle. The reason for this broadening of the time distribution is most likely the difference in time at which the particles from the cyclotron reach the target. In the cyclotron particles from more than one turn are extracted simultaneously, leading to a spread in energy. 


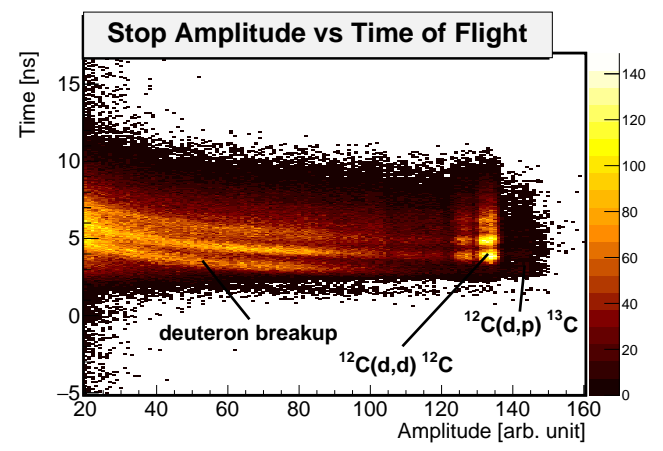

(a) Amplitude and time of flight

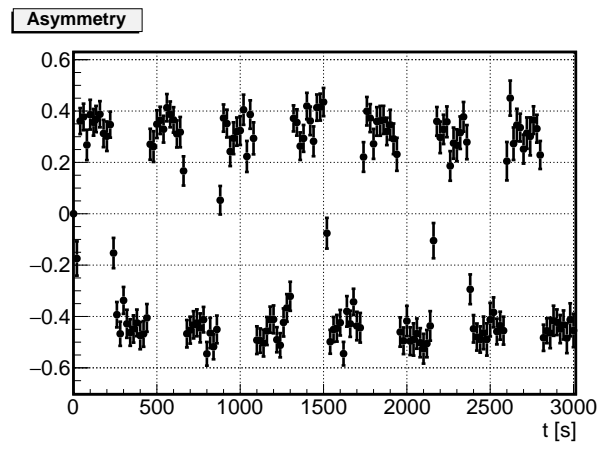

(b) Asymmetry over time.

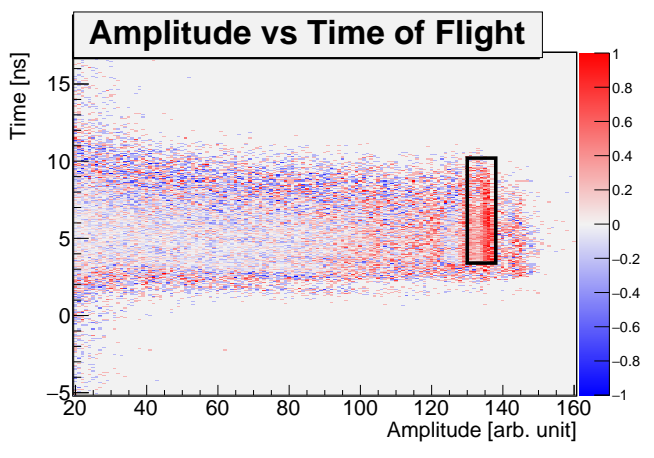

(c) Bin-by-bin asymmetry.

Figure 3: Main results of deuteron-carbon scattering experiments. All plots are taken at a scattering angle of $40^{\circ}$ using $75 \mathrm{MeV}$ deuterons scattered of a carbon target. 3a: Amplitude and time of flight. The color changes from dark brown to light yellow for increasing numbers of events. White stands for empty bins. 3b: Asymmetry $\frac{N_{L}-N_{R}}{N_{L}+N_{R}}$ over time. $N_{L, R}$ is the number of events in the left and right detector selected as shown in 3c. 3c: Bin-by-bin asymmetry. The black rectangle indicates the selected range. Red means a positive asymmetry, i.e. more particles scattered to the left than to the right, while blue indicates a negative asymmetry

Several different reactions can be identified in the spectrum, as indicated in the plot. There is a clear peak from elastic carbon scattering, the reaction that is used for polarimetry. Apart from that, there is a peak at an even higher amplitude corresponding to the reaction ${ }^{12} \mathrm{C}(\mathrm{d}, \mathrm{p}){ }^{13} \mathrm{C}$ and a broad distribution of protons from deuteron breakup. Fig. 3b shows a plot of the asymmetry between the left and the right detectors as a function of time. The two polarization states of the source are clearly separated. The statistical precision in this measurement was limited due to problems with the firmware that caused only about one in ten events to be recorded. A greater precision will be achieved in the next measurements. Fig. $3 \mathrm{c}$ is a plot of the asymmetry between the rates measured with a positive and a negative polarization in each bin for the same detector. This plot helps to identify which reactions are most suitable for polarimetry. There is a clear asymmetry in the region of elastic scattering, most other bins lie around zero.

Fig. 4 a shows a similar plot taken using a $\mathrm{CH}_{2}$-target. Fig. $4 \mathrm{~b}$ shows only those particles that arrived in coincidence with another particle at the opposite side of the detector. The recoil proton 


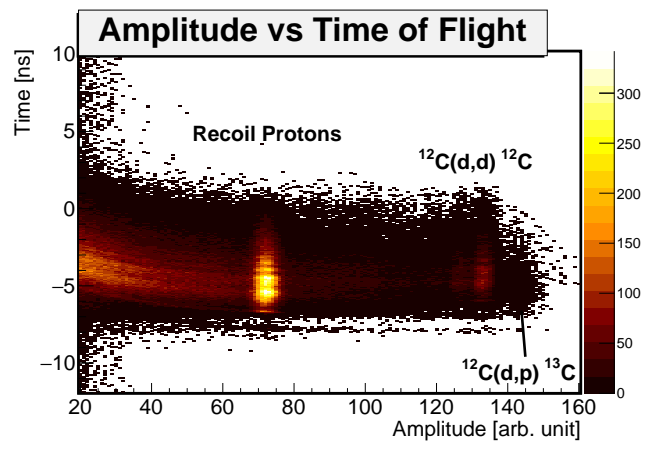

(a) Amplitude and time of flight

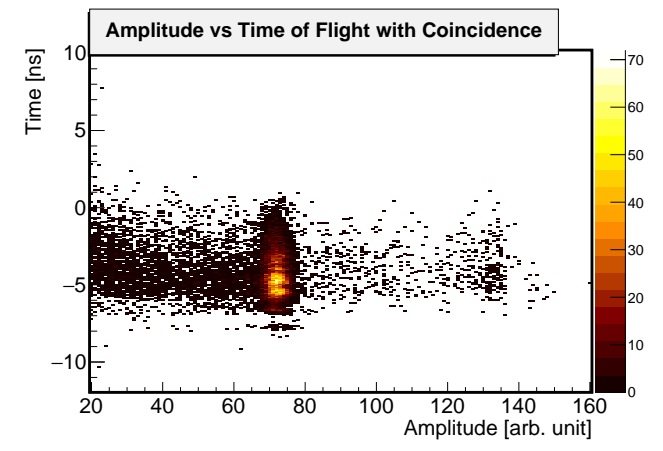

(b) Same plot using a coincidence cut

Figure 4: Main results of experiments with a $\mathrm{CH}_{2}$-target. See Fig. 3a for the color code.

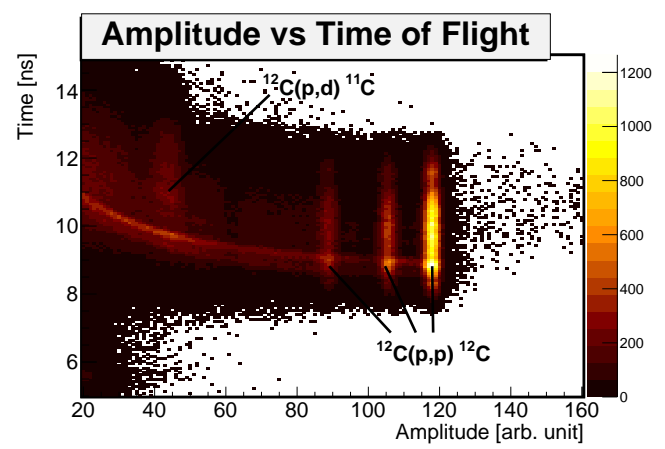

Figure 5: Amplitude and time of flight for proton carbon scattering. See Fig. 3a for the color code.

peak is almost background free in this measurement. Fig. 5 shows the time of flight and amplitude for proton carbon scattering.

\section{Conclusion and Outlook}

A new read-out for the Low Energy Polarimeter at COSY was implemented and successfully tested. In addition to the amplitude and coincidence measurements that were already possible with the old read-out, the new electronics can measure the time of flight using the cyclotron RF signal as a reference. Apart from measuring the beam polarization, the low energy polarimeter is planned to be used to determine the tensor analyzing power for deuteron scattering at $75 \mathrm{MeV}$.

\section{References}

[1] M. Eggert, Entwicklung eines gepulsten Casium-Ionenstrahls fur die Quelle polarisierter Ionen an COSY/Julich, PhD Thesis, 1998

[2] R. Maier, Nucl. Instr. and Meth. A 390 (1997) 1

[3] S. Schopferer et al. : Development and Performance Verification of the GANDALF High-Resolution Transient Recorder System, IEEE Transactions on Nuclear Science, vol.58, no.4, pp. 1456-1459, Aug. 2011 